\title{
Separation of Dopant and Mean Inner Potential Contributions to Potential Profiles Recorded from Very Highly Doped Semiconductor Layers using Electron Holography
}

\author{
David Cooper ${ }^{1}$ and Rafal E. Dunin-Borkowski ${ }^{3}$ \\ 1 CEA-LETI, 17 rue des Martyrs, F-38054 Grenoble Cedex 9, France \\ 2 Ernst Ruska-Centre for Microscopy and Spectroscopy with Electrons and Peter Grünberg Institute, \\ Forschungszentrum Jülich, D-52425 Jülich, Germany
}

Off-axis electron holography is a powerful technique for measuring electrostatic potentials in materials in the transmission electron microscope (TEM). In doped semiconductors, the characterization of dopant potentials using electron holography requires the preparation of electron-transparent specimens of highly uniform thickness, whose electrical properties are affected minimally by specimen preparation from those in the bulk device. If the specimen thickness is large enough for the effect of surface depletion on the measured phase shift to be minimal and if specimen charging and dynamical diffraction are negligible, then variations in dopant potential can in principle be measured quantitatively from a recorded phase image.

Most previous studies of dopant potentials in semiconductors using electron holography have involved the examination of specimens in which the dopant concentration is low enough (below $\sim 10^{19} \mathrm{~cm}^{-3}$ ) that local changes in dopant species or concentration could not be discerned in conventional bright-field or dark-field TEM images recorded from the same specimens. A spatial resolution of between 5 and $10 \mathrm{~nm}$ in recorded electron holograms was then also usually sufficient. However, in some of the most modern semiconductor devices, the dopant concentration may change sufficiently rapidly that a spatial resolution of approximately $1 \mathrm{~nm}$ is required in electron holographic phase images, while the dopant concentration can be sufficiently high that there may be two significant contributions to the phase change associated with the dopants in the specimen: one originating from the dopant potential of primary interest and one from the local variation in mean inner potential. The application of electron holography to such specimens requires not only the use of a highly stable microscope and a long acquisition time to measure small changes in phase with high spatial resolution but also the development of approaches to separate the dopant potential from the contribution to the measured potential associated with changes in local scattering factor and density.

Figures 1 (a) and (c) show experimental phase and amplitude images, respectively, recorded using offaxis electron holography from twelve closely-spaced very highly doped $\mathrm{B}$ layers in $\mathrm{Si}$, each of which comprises an ultra-narrow layer of dopant atoms (thinner than a few unit cells). There are two very significant observations from these images. First, the presence of dark contrast at the position of each layer in the amplitude image indicates that changes in composition and density (i.e., mean inner potential) are likely to be sufficiently strong to influence the phase image. Second, the layers are visibly wider in the phase image than in the amplitude image, suggesting that the contrast in the phase image contains a significant contribution from the dopant potential, which is expected to be wider than the compositional width of each layer. Corresponding line profiles are shown in Figs. 1 (b) and (d). The average change in phase at the position of each layer is -0.23 radians, which corresponds to a change in potential of $-0.126 \mathrm{~V}$ (using a measured value for the specimen thickness of $245 \mathrm{~nm}$ ). The average widths of the phase and amplitude profiles across the layers are 2.30 and $1.62 \mathrm{~nm}$, respectively. 
The approach that we use to interpret the dopant potential in the specimen, which is described in detail elsewhere [1], involves the independent determination of the mean inner potential contribution to the phase image shown in Fig. 1(a) by using several complementary techniques to determine the total amount of dopant, the compositional width and the local change in density at the position of each layer. The fraction of the dopant that is substitutional, which is unknown, is taken into account when comparing the predicted changed in mean inner potential with the measured potential profile [2].

\section{References:}

[1] D Cooper and R E Dunin-Borkowski, Microscopy (2013), in press.

[2] J-P Barnes, A Béché, C B Boothroyd, A Grenier, J-M Hartmann and P Rivallin are thanked for their contributions to this work.
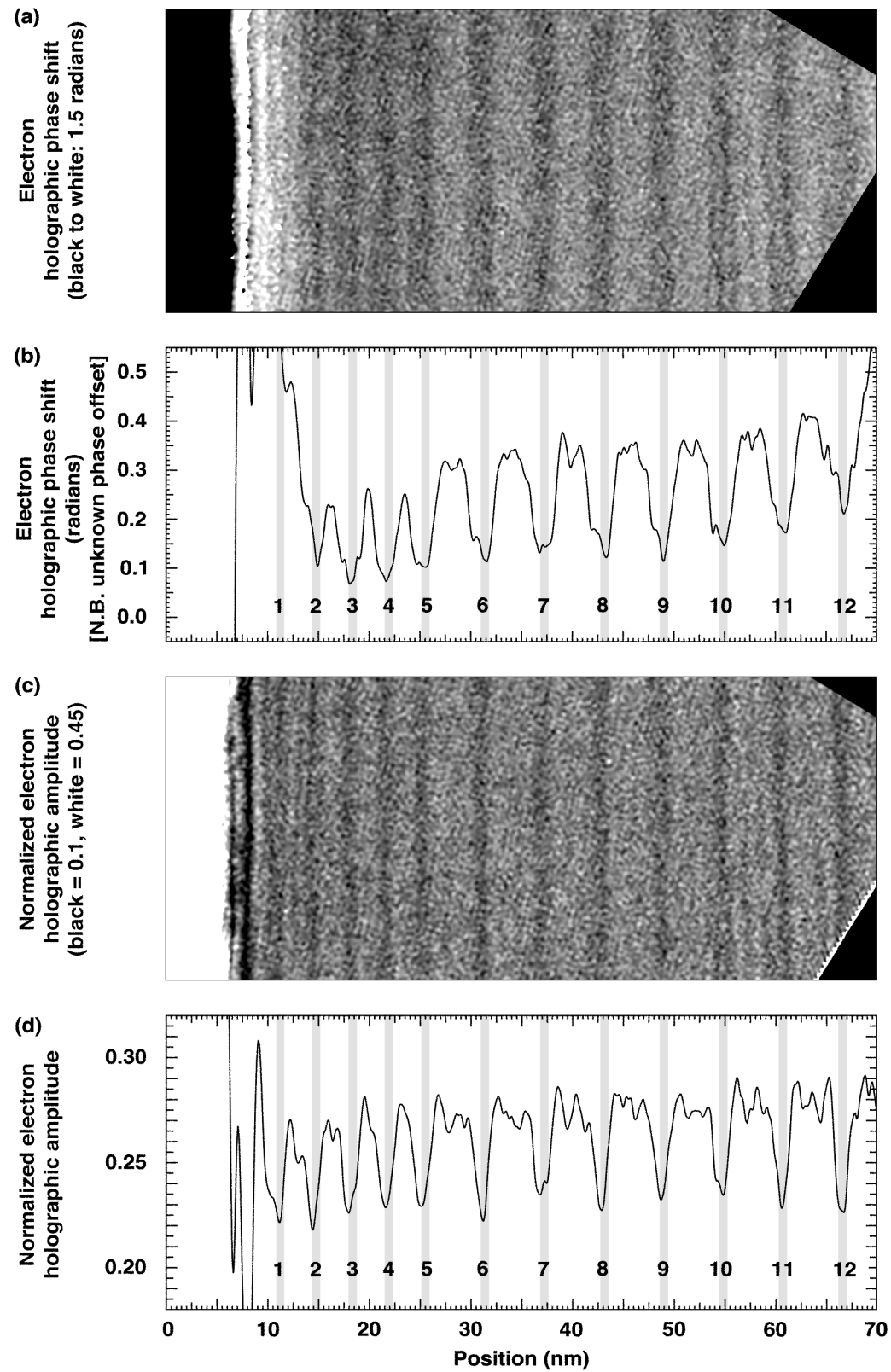

Figure 1. (a) Phase image reconstructed from an off-axis electron hologram recorded from 12 closely-spaced deltadoped $\mathrm{B}$ layers in $\mathrm{Si}$ using an interference fringe spacing of $0.33 \mathrm{~nm}$ (black to white $=1.5$ radians). (b) Line profile obtained by projecting the phase image along the layers. The vertical scale is in radians, but has an unknown additive constant as the phase in vacuum was not recorded in the same image. (c) Amplitude image reconstructed from the same off-axis electron hologram (black $=0.1$, white $=0.45$ ). (d) Line profile obtained by projecting the amplitude image along the layers. The specimen was tilted a few degrees from $<110>$ for hologram acquisition, while keeping the layers close to vertical. A vacuum reference hologram was used to remove phase distortions from the imaging and recording system of the microscope, as well as to normalize the amplitude image to a level of unity in vacuum. The layers on the right and left sides of the images show additional faint fringes, which are artifacts introduced by Fresnel fringes from the edges of the biprism wire but do not affect the line profiles significantly. The contrast from each layer is narrower in the amplitude than in the phase image. The positions of the layers are marked in grey. 